\title{
Avaliação da qualidade de vida em idosas residentes em ambientes urbano e rural
}

\section{Assessment of the quality of life of elderly women living in urban and rural environments}

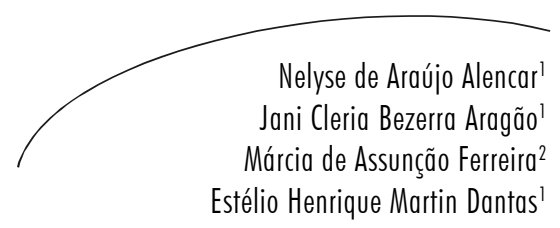

A expressão "qualidade de vida" tem sido utilizada sob diversos enfoques e é tema de pesquisas imprescindíveis na área da saúde. Muitas pesquisas têm-se dedicado a estudar a velhice sob novas óticas, mas a maioria desses estudos se refere a idosos que habitam ambientes urbanos, sendo poucas as pesquisas que abordam o tema do envelhecimento no ambiente rural. O instrumento utilizado foi o WHOQOL-OLD, modificado para pessoas idosas, com o objetivo de avaliar e comparar a qualidade de vida de idosas residentes em ambientes urbano e rural. A amostra foi constituída de 30 mulheres, com idade igual ou superior a 60 anos. O tratamento estatístico foi composto por análise descritiva e inferencial. Os dados foram analisados através SPSS, versão 13.0 para Windows. O nível de significância e erro estatístico considerado foi de $5 \%(\mathrm{p}<0,05)$. Após análise dos resultados, as idosas foram classificadas com média do Qvg-old igual ou superior a 13 como sendo detentoras de qualidade de vida suficiente; $46 \%$ das idosas residentes na área urbana apresentaram níveis iguais ou acima de 13 , sobrepondose aos $40 \%$ dos indivíduos residentes na zona rural, que obtiveram níveis superiores a 13. Constatou-se que o fato de residir em regiões geograficamente diferentes não interferiu nos níveis de qualidade de vida. Recomenda-se a realização de outros estudos, a fim de complementar e comparar os resultados de qualidade de vida com idosos de outras regiões, na perspectiva de obter uma classificação qualitativa da qualidade de vida da população idosa com diversas características sócio-demográficas e culturais.

\section{Abstract}

The term "quality of life" has been used in several approaches and is a subject of research essential for health. Much research has been dedicated to studying the old in new perspectives, but most of these studies refer to elderly who live in

\footnotetext{
Universidade Castelo Branco. Programa de Pós-Graduação Stricto Sensu em Ciências da Motricidade Humana - Procimh-UCB. Rio de Janeiro, RJ, Brasil.

2 Universidade Federal do Rio de Janeiro. Escola de Enfermagem Anna Nery. Programa de Pósgraduação e Pesquisa. Rio de Janeiro, RJ, Brasil.
}

Palavras-chave: Qualidade de Vida. População Rural. População Urbana. Idoso. Mulheres. Qualidade de vida. Ambiente rural e urbano. 
urban environments, with few researches on the subject of aging in rural areas. The instrument used was the WHOQOL-OLD, modified for the elderly, in order to evaluate and compare the quality of life of elderly residents in urban and rural environments. The sample consisted of 30 women, aged over 60 years. Statistical treatment consisted of descriptive and inferential analyses. The data were analyzed using SPSS version 13.0 for Windows. The level of error and statistical significance was set at 5\% ( $\mathrm{p}<0.05)$. After analyzing the results, the elderly were classified as medium of OQL-old less than 13 as being in possession of sufficient quality of life, $46 \%$ of elderly residents in the urban area showed levels at or above 13 , surpassing the $40 \%$ of individuals living in rural areas, which obtained levels higher than 13. It was found that the fact of living in different geographical regions not affected by levels of quality of life. It is recommended that further studies are conducted so as to complement and compare the results of quality of life of older people from other regions with a view to obtain a qualitative classification of quality of life of elderly people with various socio-demographic and cultural characteristics.
Key words: Quality of Life. Rural Population. Urban Population. Aged, Women. Quality of life; Rural and urban environments.

\section{INTRODUÇÃO}

A expressão "qualidade de vida" tem sido utilizada sob diversos enfoques e é tema de pesquisas imprescindíveis na área da saúde. Embora não haja consenso acerca da definição de qualidade de vida, esta foi definida pelo Grupo de Qualidade de Vida da Organização Mundial da Saúde como "a percepção do indivíduo de sua posição na vida, no contexto da cultura e sistema de valores nos quais ele vive e em relação aos seus objetivos, expectativas, padrões e preocupações". ${ }^{1}$

O conceito de qualidade de vida pode ser concebido como uma representação social com parâmetros objetivos - satisfação das necessidades básicas e criadas pelo grau de desenvolvimento econômico e social da sociedade - e subjetivos bem-estar, felicidade, amor, prazer e realização pessoal. Além desses parâmetros, o conceito também inclui critérios de satisfação individual e de bem-estar coletivo. ${ }^{2}$

A avaliação de qualidade de vida (QV) vem crescendo em importância como medida na avaliação de intervenções terapêuticas, de serviços e da prática assistencial cotidiana na área da saúde, e sua melhoria passou a ser um dos resultados esperados das práticas assistenciais e das políticas públicas no campo da promoção da saúde. ${ }^{3}$
A qualidade de vida de idosos tem sido foco de muitos estudos e pesquisas nas últimas décadas. Existem inúmeras iniciativas de atendimento ao idoso através de atividades diversificadas, mas pesquisas sobre os resultados dessas atividades e sua influência sobre a qualidade de vida de pessoas idosas são raramente quantificadas. ${ }^{4}$

A falta de conhecimento sobre o idoso brasileiro, a escassez de recursos humanos com competência para assisti-lo e a grande variação nas taxas de envelhecimento populacional nas diferentes regiões brasileiras fazem com que a transposição das disposições dos direitos do idoso para o cotidiano da população seja um dos grandes desafios para os próximos anos e dependa do contexto regional a qual ele se insere. ${ }^{5}$ Neste sentido, muitos estudos têm-se dedicado a estudar a velhice sob novas óticas, mas a maioria deles se refere a idosos que habitam em ambientes urbanos, sendo poucas as pesquisas que abordam o tema do envelhecimento no ambiente rural.

Caracteriza-se o ambiente rural os municípios que possuem menos de 25.000 habitantes. Aproximadamente 75\% dos municípios brasileiros se enquadram nesta deûnição, e neles residem cerca de 40 milhões de pessoas. Esses municípios rurais são constituídos por um núcleo urbano, que corresponde à sede municipal, e um núcleo 
agrário, onde se desenvolvem atividades de agricultura e pecuária. ${ }^{6}$

A ausência de um instrumento que avaliasse qualidade de vida, com uma perspectiva internacional, fez com que a OMS constituísse um Grupo de Qualidade de Vida (Grupo WHOQOL) com a finalidade de desenvolver instrumentos capazes de fazê-lo dentro de uma perspectiva transcultural. ${ }^{1}$ Inicialmente, foi desenvolvido o World Health Organization Quality of Life (WHOQOL-100 e o WHOQOL-BREF ).

O instrumento WHOQOL-100 consiste em cem perguntas referentes a seis domínios: físico, psicológico, nível de independência, relações sociais, meio ambiente e espiritualidade/ religiosidade/ crenças pessoais. O WHOQOLBREF consta de 26 questões, é multidimensional e avalia a qualidade de vida geral; com quatro domínios maiores: saúde física, saúde psicológica, relações sociais e meio ambiente.

O recordatário a ser utilizado nesta pesquisa será o WHOQOL-OLD ${ }^{7}$, modificado para pessoas idosas, que avalia a qualidade de vida através de seis Domínios ou facetas:

Domínio I - "Funcionamento do Sensório" (FS) - avalia funcionamento sensorial e o impacto da perda das habilidades sensoriais nas atividades da vida diária e da capacidade de interação com outras pessoas na qualidade vida de idosos.

Domínio II - "Autonomia” (AUT) - Refere-se a independência na velhice, descrevendo até que ponto se é capaz de viver de forma autônoma e tomar suas próprias decisões.

Domínio III - "Atividades Passadas, Presentes e Futuras" (PPF) - refere-se a atividades passadas, presentes e futuras, descrevendo a satisfação sobre conquistas na vida e projetos anseios futuros.

Domínio IV - "Participação Social" (PSO) refere-se à participação social, que delineia a participação em atividades do quotidiano, especialmente na comunidade em que se está inserido.
Domínio V - "Morte e Morrer" (MEM) - está relacionada às preocupações, inquietações, expectativas e temores sobre a morte e morrer.

Domínio VI - "Intimidade" (INT) - refere-se à intimidade, que avalia a capacidade de ter relações pessoais e íntimas.

Buscou-se, então, analisar a qualidade de vida, comparando os resultados da avaliação, em idosas residentes em ambiente urbano e rural, cadastradas em Unidades Básicas da Estratégia Saúde da Família dos municípios do Juazeiro do Norte e Crato-CE, Brasil.

\section{MATERIAIS E MÉTODOS}

A pesquisa caracterizou-se como quaseexperimental do tipo ex post facto, descritiva e comparativa. As participantes apresentavam idade igual ou superior a 60 anos, sorteada aleatoriamente do universo de 120 idosas, perfazendo no final um total de 30, cadastradas em duas unidades de Saúde da Família das zonas urbana e rural das cidades de Juazeiro do Norte e Crato- CE, respectivamente.

A amostra foi caracterizada como finita nãoprobabilística intencional e escolhida por conveniência, mediante aceitação em participar do estudo, através da assinatura do Termo de Consentimento Livre e Esclarecido, registrada sob protocolo número 0159/2008 do Comitê de Ética e Pesquisa da Universidade Castelo Branco-RJ, de acordo com a Resolução CONEP nº 196/96.

Como critério de inclusão, as participantes deveriam ser cadastradas nas unidades do Programa Saúde da Família dos referidos municípios, ser do sexo feminino e ter idade igual ou superior a 60 anos.

Foram considerados critérios de exclusão: indivíduos do sexo masculino, e/ou a idosa com qualquer tipo de enfermidade aguda ou crônica que pudesse comprometer ou se tornar um fator de impedimento para os testes realizados, as que faziam uso de medicamentos que causassem distúrbios de atenção ou as não cadastradas nas respectivas unidades de saúde. 
A qualidade de vida das idosas foi avaliada por meio do questionário WHOQOL-OLD, que contém seis facetas (ou domínios) de quatro itens cada (o módulo consiste em 24 itens) avaliados pela da escala de Likert (1 a 5 pontos) atribuídos a seis facetas. Para todas as facetas, o escore dos valores possíveis pode oscilar de 4 a 20, desde que todos os itens de uma faceta tenham sido preenchidos. Os escores destas seis facetas ou os valores dos 24 itens do módulo WHOQOL-OLD podem ser combinados para produzir um escore geral (QVG-OLD) para a qualidade de vida em adultos idosos, denotado como o "escore total" do módulo WHOQOLOLD. Basicamente, escores altos representam uma alta qualidade de vida e escores baixos representam uma baixa qualidade de vida.

O questionário foi aplicado individualmente e foi solicitado aos indivíduos, ao responderem, que tivessem em mente os próprios valores, aspirações, prazeres e preocupações, tomando por base as duas últimas semanas.

A análise dos dados foi realizada utilizando-se o pacote estatístico SPSS, versão 13.0 para Windows. Os resultados da análise estatística descritiva são apresentados com média, desvio padrão, mínima e máxima e a análise estatística inferencial foi desempenhada pelo teste $t$-student, com o objetivo de verificar a existência de diferenças significativas entre os testes em ambos os grupos. Para todos os procedimentos, adotouse um intervalo de confiança de $5 \%(\mathrm{p}<0,05)$.

\section{RESULTADOS}

A tabela 1, a seguir, apresenta a estatística descritiva e inferencial para os resultados da qualidade de vida do grupo de idosas residentes na zona urbana (U) e rural (R) dos municípios citados.

Tabela 1 - Estatística descritiva e inferencial da qualidade de vida (WHOQOL-OLD) em idosas residentes da zona urbana e rural. Juazeiro do Norte e Crato, CE.

\begin{tabular}{lrllrrrrrr}
\hline \multirow{2}{*}{ Domínios } & \multicolumn{2}{c}{ média } & \multicolumn{2}{c}{$\mathrm{dp}$} & \multicolumn{2}{c}{ mín } & \multicolumn{2}{c}{ máx } & $\mathrm{P}$ \\
\cline { 2 - 9 } & $\mathrm{U}$ & $\mathrm{R}$ & $\mathrm{U}$ & \multicolumn{1}{c}{$\mathrm{R}$} & $\mathrm{U}$ & \multicolumn{1}{c}{$\mathrm{R}$} & $\mathrm{U}$ & $\mathrm{R}$ & \\
\hline Dom 1 & 9,7 & 10,7 & 2,4 & 2,6 & 6,0 & 6,0 & 15,0 & 15,0 & 0,29 \\
Dom 2 & 13,6 & 10,5 & 3,6 & 3,8 & 7,0 & 5,0 & 18,0 & 16,0 & 0,03 \\
Dom 3 & 13,8 & 11,8 & 2,4 & 2,2 & 9,0 & 7,0 & 18,0 & 16,0 & 0,02 \\
Dom 4 & 15,4 & 13,9 & 2,2 & 2,4 & 12,0 & 9,0 & 9,0 & 18,0 & 0,09 \\
Dom 5 & 12,6 & 14,3 & 4,4 & 11,9 & 4,0 & 10,0 & 18,0 & 4,0 & 0,17 \\
Dom 6 & 14,5 & 14,4 & 3,7 & 1,6 & 4,0 & 10,0 & 20,0 & 17,0 & 0,95 \\
QVG -OLD & 13,2 & 12,6 & 1,9 & 0,3 & 11,6 & 10,0 & 16,3 & 15,0 & 0,19 \\
\hline
\end{tabular}

A=ativas, $\mathrm{S}=$ sedentárias, $\mathrm{dp}=$ desvio padrão, mín=mínima, max=máxima, Dom1=Funcionamento do sensório, Dom2= Autonomia, Dom3=Atividades Passadas, Presentes e Futuras, Dom4=Participação Social, Dom5=Morte e Morrer, Dom6=Intimidade, Qvg-old=qualidade de vida geral.

\section{DISCUSSÃO}

Ao analisar os dados da tabela 1, classificamos as idosas com média do Qvg-old igual ou superior a 13 como sendo detentoras de qualidade de vida suficiente, enquanto aquelas que apresentaram condições inferiores foram classificadas com Qvg-old insuficiente. Tomamos como base para esses resultados os estudos realizados por $\mathrm{Mello}^{4}$, ao utilizar o mesmo instrumento em idosos, apresentando a média para QV semelhante ao encontrado na presente pesquisa $(\mathrm{QV}$ média $=13)$. A mesma propõe uma classificação categórica do WHOQOL-OLD, onde escores entre 14,1 e 20 correspondem a QV alta, entre 11 e 14 a QV, média, e escores abaixo de 10,9 significam QV baixa. 
Observou-se, na amostra, que $46 \%$ da idosas residentes na área urbana apresentaram níveis iguais ou acima de 13 , sobrepondo-se às $40 \%$ das idosas residentes na zona rural, que obtiveram níveis superiores a 13. Esses dados estão muito aquém dos resultados obtidos por Mello ${ }^{4}$, que apresentou um escore total de QV próximo a $60 \%$ e da pesquisa realizada por Hawthorne ${ }^{8}$, que examinou a qualidade de vida de idosos através do instrumento WHOQOL-Bréf, encontrando escore total de $70 \%$.

Analisando-se separadamente os domínios de cada grupo, observa-se que os menores escores foram os dos domínios $1(\mathrm{U}=9,7)$ e $2(\mathrm{R}=10,5)$, e os maiores escores dos domínios $4(\mathrm{U}=15,4)$ e 6 $(\mathrm{R}=14,4)$. Tal fato corrobora o estudo de Mello ${ }^{4}$, que observou o menor escore na faceta $1(9,94) \mathrm{e}$ o maior escore na faceta $4(15,11)$.

Pereira et. al. ${ }^{9}$ investigaram a contribuição de cada domínio da qualidade de vida (físico, social, psicológico e ambiental) na qualidade de vida global, de 211 idosos, residentes no município de Teixeiras, na Região Sudeste do Brasil, através do WHOQOL- Bref. Os quatro domínios (físico, psicológico, ambiental e social) juntos explicam $36,1 \%$ da qualidade de vida global. O domínio social tem pequena contribuição $(0,4 \%)$, mas não significativa ( $\mathrm{p}$ $>0,05)$. O domínio que mais contribui na qualidade de vida global é o físico $(28,2 \%)$, seguido do ambiental $(6,2 \%)$ e do psicológico $(1,3 \%)$. Embora os domínios isoladamente não possam ser utilizados como parâmetro de comparação com as facetas do WHOQOLOLD, o escore total de qualidade de vida apresenta-se muito baixo, se comparado ao estudo citado, que encontrou um escore total de $65 \%$ de qualidade de vida.

Observa-se que o fato de residir em perímetros urbanos acarreta, em alguns domínios, melhores escores. Isso ocorre, possivelmente, pelo fato de as idosas do meio urbano possuírem melhores níveis econômicos, o que facilitaria maior acesso e acessibilidade a meios de comunicação, saúde, lazer, aquisição de bens de consumo, dentre outros.
Constatou-se nesta amostra, entretanto, que o fato de residir em áreas geograficamente diferentes não esteve associado a melhores níveis de qualidade de vida ( $p>0,01$ em todos os domínios), o que pode nos levar a questionar que talvez a $\mathrm{QV}$ possa sofrer influências de outros fatores, como o baixo poder aquisitivo, observado na maioria das residentes da zona rural estudada. Todavia, essa variável não foi avaliada na presente pesquisa, mas estudos realizados por Trentini ${ }^{10}$ confirmaram que idosos de nível econômico mais baixo apresentaram os piores índices de qualidade de vida em todos os domínios avaliados.

Os resultados desta pesquisa contrariam o estudo de Sequeira \& Silva ${ }^{11}$, realizado em Portugal, que concluiu que o ambiente rural oferece melhores condições para o envelhecimento do que o meio urbano. Provavelmente, as condições materiais daquele país permitam subsidiar condições melhores de qualidade de vida a seus idosos.

Em pesquisa semelhante, analisando a QV de idosos do meio urbano e rural através do WHOQOL-BREF, Martin et. $\mathrm{al}^{6}$ encontraram escores inferiores (QV média $=3,46)$ aos da presente pesquisa, não encontrando também diferenças estatísticas significantes ao comparar os dois grupos.

Como o WHOQOL-OLD é um instrumento recente específico para o idoso, e as publicações que utilizam esse instrumento ainda são restritas, sobretudo quando se avalia a qualidade de vida dessa população em regiões demográficas diferentes, impossibilitando uma comparação mais específica com outras pesquisas. Baseado nisso, foi realizada análise comparativa levando em consideração somente o escore total (QVG) dos instrumentos WHOQOL-OLD e BREF.

Pereira \& colaboradores ${ }^{9}$ verificaram as características biofísicas de mulheres idosas na Barra da Tijuca, região de classe média alta do Rio de Janeiro. Em relação à qualidade de vida (QVG), o instrumento usado nesse estudo foi o WHOQOL-100 (OMS, 1998). O escore de QVG foi $15,57 \pm 1,40$. Mello \& Morucci ${ }^{12}$ também identificaram a qualidade de vida de idosos 
utilizando o WHOQOL-100, com 17 idosos, de ambos os gêneros, que praticam exercícios físicos regularmente, na Barra da Tijuca, zona da cidade do Rio de Janeiro cuja média da qualidade de vida foi 14,17 .

Varejão \& colaboradores ${ }^{13}$, em estudo sobre a qualidade de vida de idosos residentes em Jacarepaguá, no Rio de Janeiro, através do WHOQOL-100, observaram os escores das facetas, variando de 8,21 a 15,47.

Comparando os escores obtidos junto à população do presente estudo, observa-se que os valores de QVG-OLD encontrados assemelhamse aos níveis de QV global das pesquisas citadas.

\section{CONCLUSÃO}

$\mathrm{Na}$ maioria das vezes, as pesquisas científicas se pautam na busca de constatar as diferenças entre os fenômenos estudados. Este estudo também partiu deste pressuposto, pois eram esperados resultados diferenciadores entre os níveis de QV no ambiente rural e urbano. No entanto, a presente pesquisa pôde inferir que o fato de residir em áreas geograficamente diferentes não interferiu nos índices de qualidade de vida dessa população.
A falta de tal diferenciação aponta possivelmente para um aspecto importante: o fato de que as pessoas, independentemente de residirem em cidades grandes ou pequenas, em centros urbanos ou no interior do país, podem sentir-se bem com suas vidas, e que a percepção sobre qualidade de vida possa ser bem mais subjetiva e inerente ao intrínseco pessoal do que estar relacionada a fatores externos como o ambiental ou o sócio-econômico.

Ressaltamos que a importância de estudos sobre as condições e determinantes de saúde dos idosos são fundamentais para subsidiar políticas de saúde voltadas a essa população.

Recomenda-se a realização de outros estudos a fim de complementar e comparar os resultados de qualidade de vida, obtidos no presente estudo, com outros grupos de idosos de outras regiões, com a perspectiva de obter uma classificação qualitativa da qualidade de vida da população idosa com diversas características sóciodemográficas e culturais.

\section{AGRADECIMENTOS}

Agradeço às idosas participantes do estudo e aos professores orientadores da pesquisa.

\section{REFERÊNCIAS}

1. Fleck MPA, et al. Aplicação da versão em português do instrumento abreviado de avaliação da qualidade de vida “WHOQOL-bref”. Rev Saúde Pública 2000 [acesso em 2007 Jan 20]; 34(2). Disponível em: URL: http://www.scielo.br/scielo.php?pid= S003489102000000200012\&script=sci_arttext

2. Minayo MCS, Hartz ZMA, Buss PM. Qualidade de vida e saúde: um debate necessário. Ciên Saúde Colet 2000 [acesso em 2007 Mar 09];

5(1). Disponível em: URL: http:// www.scielo.br/scielo.php

3. Bittencourt ZZLC; Hoehne EL. Qualidade de vida de deficientes visuais. Medicina, Ribeirão Preto 2006 abr./jun [acesso em 2007 Mar 09]; 39(2): 260-4. Disponível em: URL: <http// :www.fmrp.usp.br/revista/2006/vol39n2/ ao_qualidade_vida_deficientes_visuais1.pdf $>$.

4. Mello DB. Influência da obesidade na qualidade de vida de idosos. [tese]. Rio de Janeiro: Fundação Oswaldo Cruz. Programa de doutorado em Ciências na área de Saúde Pública da Fundação Oswaldo Cruz, Escola Nacional de Saúde Pública Sergio Arouca; 2008.

5. Veras R. Em busca de uma assistência adequada à saúde do idoso: revisão de literatura e aplicação de um instrumento de detecção precoce e de previsibilidade de agravos. Caderno de Saúde Pública 2003; 19(3): 705-15.

6. Martins CR, et al. Avaliação da qualidade de vida subjetiva dos idosos: uma comparação entre os residentes em cidades rurais e urbanas. Estudos Interdisciplinares sobre o Envelhecimento 2007; 11: 135-54.

7. Fleck MPA, Chachamovich E, Trentini CM. WHOQOL-OLD Project: method and focus 
group results in Brazil. Revista Saúde Pública 2003 [acesso em 2007 Mar 09], 37(6): 793-9. Available from: URL: http://www.scielosp.org/scielo.php

8. Hawthorne $\mathrm{G}$, et al. Issues in conducting crosscultural research: implementation of an agreed international protocol [corrected] designed by the WHOQOL Group for the conduct of focus groups eliciting the quality of life of older adults. Qual Life Res 2006 sep; 15(7): 1257-70.

9. Pereira RF, et al. Contribuição dos domínios físico, social, psicológico e ambiental para a qualidade de vida global de idosos. Revista de Psiquiatria 2006 abr; 28(1): 27-38.

10. Trentini CM. Qualidade de vida em idosos: a construção de uma escala de qualidade de vida para idosos - OMS. 2004. 224f. [tese]. Porto Alegre: Programa de Pós-Graduação em Ciências Médicas: Psiquiatria, Faculdade de Medicina, Universidade
Federal do Rio Grande do Sul; 2004.

11. Sequeira A, Silva MN. O bem estar da pessoa idosa no meio rural. Análise Psicológica 2002; 20 (3): 50516.

12. Mello DB, Morucci C. Avaliação da qualidade de vida de Idosos praticantes de exercícios regulares. Trabalho de Conclusão de Curso de Educação Física. Universidade Estácio de Sá, Rio de Janeiro; 2005.

13. Varejão RV, Dantas EHM, Matsudo SMM. Comparação dos efeitos do alongamento e do flexionamento, ambos passivos, sobre os níveis de flexibilidade, capacidade funcional e qualidade de vida do idoso. Revista Brasileira de Ciência em Movimento 2007; 15(2): 87-95.

Recebido: 05/5/2009

Aprovado: 21/9/2009 
